\title{
Experiencias
}

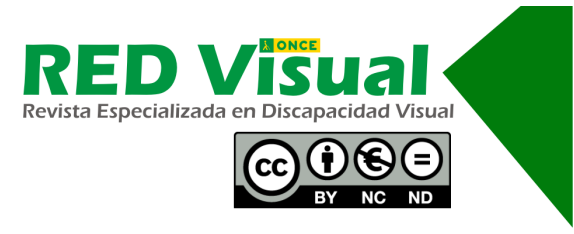

\section{Recursos metodológicos que favorecen la intervención comunicativa con niños con sordoceguera utilizando la realidad aumentada: libro de comunicación Yo soy ${ }^{1}$}

\author{
Resource favouring communicational intervention \\ with deafblind children using augmented reality: Yo soy [I am], \\ introduction to communicational methodology
}

\section{González Donate}

\section{Resumen}

La sordoceguera no es una simple suma de discapacidades, sino que supone en mayor o menor medida aislamiento e incomunicación. Es el entorno y no el niño con sordoceguera el que tiene que adaptarse para minimizar el impacto de esta discapacidad y este llegue a conseguir la mayor calidad de vida posible. La figura del adulto es la que da sentido a las producciones del niño y favorece la relación comunicativa. Es necesario crear recursos metodológicos personalizados, ya que cada niño tiene una forma única de ser y estar en el mundo, su comunicación es particular y es la manifestación de su historia de relación con el entorno. El libro Yo soy es un libro de presentación que se dirige al entorno de niños con sordoceguera congénita que presentan una comunicación muy básica y no alcanzan motivación cognitiva. Se situarían en el primer nivel de intervención: la interacción comunicativa. Es un libro físico, fácil de utilizar, que puede acompañar al niño en todo momento y que, a través de la realidad aumentada con códigos QR, nos lleva a información virtual, en este caso a vídeos de comunicación con lengua de signos apoyada, ayudando a la eliminación de las barreras de comunicación y favoreciendo las experiencias comunicativas. En el artículo

\footnotetext{
1 Trabajo galardonado con el Segundo Premio en el IV Concurso de Experiencias de Innovación y Buenas Prácticas en Servicios Sociales de la ONCE, presentado con el título Elaboración de recursos metodológicos que favorecen la intervención comunicativa con niños sordociegos utilizando la realidad aumentada: libro de comunicación Yo soy.
}

González, M. (2020). Recursos metodológicos que favorecen la intervención comunicativa con niños con sordoceguera utilizando la realidad aumentada: libro de comunicación Yo soy. RED Visual: Revista Especializada en Discapacidad Visual, 76, 24-33. https://doi.org/10.53094/MBMV7925. 
se muestran los pasos a seguir para su creación e inclusión de la realidad aumentada en el mismo, así como los resultados tan satisfactorios del uso del libro en un caso concreto en los distintos entornos.

\title{
Palabras clave
}

Sordoceguera congénita. Interacción comunicativa. Libro de presentación. Realidad aumentada.

\begin{abstract}
More than the mere sum of two disabilities, deafblindness entails, to a greater or lesser extent, isolation and uncommunication. It is the environment, not the deafblind child, that must adapt to minimise the impact of that disability enabling those affected to attain the highest quality of life possible. The adult figure endows the child's output with meaning and favours communicational relationships. Personalised methodological resources are required, for each child has a unique way of being and their specific approach to communication is a window on the history of their relations with their surrounds. Yo soy is an introductory book intended for those close to congenitally deafblind children with very basic communication skills. Failing to attain cognitive motivation, they would be positioned on the first level of intervention: communicative interaction. This printed, easy-to-use book is geared to continuous use with the child. Through QR code-based augmented reality, it features virtual information, in this case videos and supplementary sign language, that lower communication barriers and favour communicational experience. The article sets out the steps to be followed for the creation and inclusion of the augmented reality described, and provides an account of the highly satisfactory results of the use of the book in a specific case in different environments.
\end{abstract}

\section{Key words}

Congenital deafblindness. Communicative interaction. Introductory book. Augmented reality.

\section{Presentación y justificación}

Este proyecto tiene el propósito de crear una estrategia metodológica para la intervención en el primer nivel del desarrollo de la comunicación: la interacción comunicativa en los niños con sordoceguera congénita. La creación e introducción de estrategias metodológicas viene recogida dentro de las directrices generales para la

González, M. (2020). Recursos metodológicos que favorecen la intervención comunicativa con niños con sordoceguera utilizando la realidad aumentada: libro de comunicación Yo soy. RED Visual: Revista Especializada en Discapacidad Visual, 76, 24-33. https://doi.org/10.53094/MBMV7925. 
atención a afiliados a la ONCE con sordoceguera: «Introducir y homogeneizar criterios metodológicos y de atención de acuerdo a las directrices de la UTS» (Unidad Técnica de Sordoceguera de la ONCE, 2010).

Los niños con sordoceguera congénita se enfrentan a una dificultad enorme por su limitación en el conocimiento de lo que existe y pasa en el entorno.

Por pocas conductas comunicativas que manifieste el niño, el adulto le responde y les da sentido. Tiene que sentir que se le escucha. El carácter de diálogo, incluso de las interacciones tempranas, está basado en la interpretación del adulto de la conducta observada; el niño necesita experimentar que su comportamiento tiene un efecto a su alrededor. En este sentido, la observación del comportamiento del niño, atendiendo a cualquier detalle que pueda ser entendido como comunicativo, así como la consistencia de la respuesta del adulto son fundamentales.

En este primer nivel de intervención en comunicación se trabaja sobre la interacción, favoreciendo la conexión del niño con el entorno, facilitando las experiencias compartidas significativas, desarrollando todo su potencial global y la inclusión en todos sus entornos (familiar, escolar y social).

De ahí la importancia de crear, desde las primeras etapas, estrategias metodológicas educativas, para intervenir en la interacción comunicativa desde el enfoque de Jan Van Dijk (1968) - profesor holandés con larga experiencia en niños con sordoceguera congénita-, dirigidas a:

- Establecer una «relación de confianza».

- Estructurar la jornada diaria para hacer previsible lo que va a ocurrir. La organización de la actividad diaria debe de atender al espacio, el tiempo y a las personas (Gómez, 2017).

Partiendo de estas premisas, se diseña el libro de comunicación Yo soy.

¿Por qué un libro de comunicación? Porque es un recurso que facilita la comunicación entre el niño y su entorno, en el que se explican los sistemas y las estrategias comunicativas más adecuados en la relación con el niño. Es un libro físico, fácil de utilizar, que puede acompañar al niño con sordoceguera en todo momento, que re-

González, M. (2020). Recursos metodológicos que favorecen la intervención comunicativa con niños con sordoceguera utilizando la realidad aumentada: libro de comunicación Yo soy. RED Visual: Revista Especializada en Discapacidad Visual, 76, 24-33. https://doi.org/10.53094/MBMV7925. 
copila fotos, vídeos, textos, códigos QR, pictogramas..., y que puede ser utilizado por todo su entorno. Es un material que permite la personalización según las necesidades de cada niño con sordoceguera.

¿Por qué utilizar la realidad aumentada?

La definición de realidad aumentada según la Wikipedia:

La realidad aumentada (RA) es el término que se usa para describir al conjunto de tecnologías que permiten que un usuario visualice parte del mundo real a través de un dispositivo tecnológico con información gráfica añadida por este. El dispositivo, o conjunto de dispositivos, añaden [sic] información virtual a la información física ya existente, es decir, una parte virtual aparece en la realidad. De esta manera los elementos físicos tangibles se combinan con elementos virtuales, creando así una realidad aumentada en tiempo real. ${ }^{2}$

Para este proyecto se utiliza la realidad aumentada de nivel 0: códigos QR. Hoy en día todo el mundo tiene un dispositivo móvil y es fácil descargarse una aplicación gratuita de lector de códigos QR que enlaza estos códigos insertados en el libro físico, en este caso, a vídeos reales.

\section{Objetivos}

\subsection{Objetivos generales}

- El objetivo prioritario para la intervención comunicativa con niños con sordoceguera es favorecer que el entorno elimine en lo posible las limitaciones de esta discapacidad, ayudándoles en su inclusión en la sociedad en la que viven con el propósito claro del desarrollo de la comunicación dentro de las peculiaridades y competencias de cada alumno.

Para ello se propone el enfoque ambiental de la discapacidad que surgió de las normas de las Naciones Unidas. Este enfoque indica que es el entorno, y no la persona con discapacidad, lo que debe cambiar para minimizar el impacto de una discapacidad (Rodbroe y Janssen, 2014). Es fundamental la figura del adulto que pueda consolidar y reforzar la utilización conjunta de la adaptación táctil de

2 https://es.wikipedia.org/wiki/Realidad_aumentada.

González, M. (2020). Recursos metodológicos que favorecen la intervención comunicativa con niños con sordoceguera utilizando la realidad aumentada: libro de comunicación Yo soy. RED Visual: Revista Especializada en Discapacidad Visual, 76, 24-33. https:/doi.org/10.53094/MBMV7925. 
la lengua de signos u otras adaptaciones que favorezca un desarrollo comunicativo más acorde a las características del niño con sordoceguera. Es necesario un compañero de comunicación competente (Rodbroe y Janssen, 2014) que sea capaz de aumentar el interés del niño por participar en experiencias compartidas que le lleven a negociar significados, aumentar el vocabulario, favorecer de forma natural a lo largo del día el desarrollo de la comunicación y le ayude a comprender todas las situaciones de su entorno.

- Junto al objetivo prioritario, este recurso pretende potenciar el desarrollo de la comunicación en el primer nivel y conocer la forma única de comunicarse de cada niño con sordoceguera, así como entender qué es la manifestación de su historia de relación biológica, emocional, cognitiva y social establecida.

\subsection{Objetivos específicos}

- Ofrecer al entorno que se relaciona con los niños con sordoceguera pautas y orientaciones que favorezcan las interacciones comunicativas.

- Fomentar una relación de confianza en un entorno organizado y predecible.

- Desarrollar conductas preverbales de comunicación orientadas a la petición.

- Diseñar recursos educativos para trabajar las distintas estrategias metodológicas.

\section{Población}

El libro Yo soy, es un libro de presentación que se dirige a niños con sordoceguera congénita que presentan dificultades o en los que están en desarrollo la interacción comunicativa, la comunicación receptiva y la expresiva y que tienen un nivel bajo de funcionamiento en la comunicación que queda limitado a aspectos básicos, por no alcanzar «la motivación cognitiva».

Este material está pensado para facilitar la comunicación entre el alumno y su entorno familiar, escolar y social, así como la coordinación y el trabajo en red, a través de la explicación de los sistemas y estrategias más adecuados en función de las necesidades comunicativas. Se ha creado para establecer una relación positiva y de confianza con

González, M. (2020). Recursos metodológicos que favorecen la intervención comunicativa con niños con sordoceguera utilizando la realidad aumentada: libro de comunicación Yo soy. RED Visual: Revista Especializada en Discapacidad Visual, 76, 24-33. https://doi.org/10.53094/MBMV7925. 
los adultos, pero también se podría realizar un libro de gestos y signos para esta misma estrategia y un libro para trabajar la creación de sintonía y corregulación con juegos de interacción conjunta de estructura circular también llamados de maternaje. Así mismo, se podrían crear libros de rutinas y de calendarios de anticipación para establecer un orden y una estructura en la actividad diaria, siempre utilizando la realidad aumentada.

\section{Temporalización}

Antes de la realización del libro se lleva a cabo una reunión con las personas de los distintos entornos para explicar el recurso a realizar, con sus objetivos, justificación y metodología a seguir. El primer paso es observar al niño con sordoceguera en los distintos ambientes y las distintas interacciones que realiza con los adultos, con los objetos, con los compañeros, con los hermanos, etc. Se utiliza la escala Callier-Azusa (H) (Stillman y Battle, 1985) y la Matriz de Comunicación de Charity Rowland (Rowland, 1990). Una vez recogida la información, hay que estructurarla y darle forma para volver a reunirse y ver las necesidades comunicativas a trabajar, la realización de las fotos y vídeos, decidir la plantilla más adecuada y el software a emplear para el diseño del libro físico. Posteriormente, se entrega una propuesta bastante ajustada de los pasos a seguir en la creación del libro y se realizan los ajustes que sean necesarios.

El libro de comunicación Yo soy, que se presenta como ejemplo, se realizó el curso pasado. En el primer trimestre se realizaron sesiones de observación de la niña y varias reuniones con el centro escolar y la familia con el objetivo de recabar toda la información y decidir las necesidades comunicativas a trabajar. Los aspectos básicos elegidos a plasmar en el libro fueron la presentación, la utilización de la lengua de signos apoyada para la comunicación receptiva, el juego para entrar en la motivación de la niña, la anticipación y la despedida. A partir del segundo trimestre la niña lo llevaba siempre consigo en la barra de la silla de ruedas en la que se desplazaba, de tal manera que las personas que se relacionaban con la niña lo podían consultar en todo momento.

\section{Proceso de creación del libro de comunicación Yo soy}

Las fases a seguir para la creación de un libro de comunicación son las siguientes:

1. Creación de plantilla en Word para la maquetación del libro de comunicación.

González, M. (2020). Recursos metodológicos que favorecen la intervención comunicativa con niños con sordoceguera utilizando la realidad aumentada: libro de comunicación Yo soy. RED Visual: Revista Especializada en Discapacidad Visual, 76, 24-33. https://doi.org/10.53094/MBMV7925. 
Figura 1. Plantilla del libro de comunicación

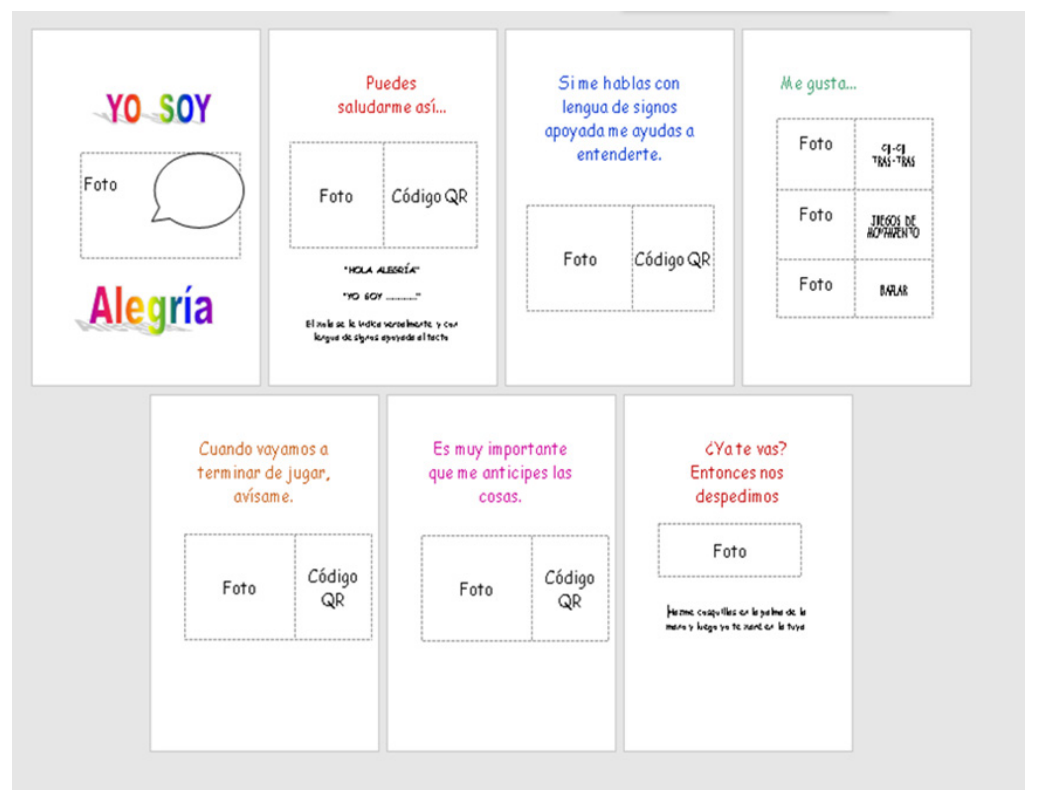

2. Desarrollo de los elementos de la realidad aumentada a emplear, con la explicación para ser aplicados en la elaboración del libro.

a. Realizar los vídeos y fotos con cualquier dispositivo (cámara, móvil, etc.) y guardar los mismos en el ordenador.

b. Crear un canal de YouTube privado y subir los vídeos, pixelando las caras de las personas que están en él para proteger su intimidad.

b.1. Para crear una cuenta en YouTube es necesario tener una cuenta de correo electrónico en Gmail.

b.2. Subir el vídeo de un archivo del ordenador e indicar la categoría de «Oculto», con el objetivo de que sea visible solamente para aquellas personas que tengan acceso al mismo.

b.3. Una vez subido el vídeo, indicar un «Nombre» y una «Descripción» y, cuando termine de procesarlo al $100 \%$, hacer clic en «Listo».

b.4. Se genera la URL del vídeo de YouTube que luego utilizaremos para la creación del código QR. 
b.5. Editar el vídeo en YouTube para pixelar las caras de las personas que aparecen en él. En la opción de «Edición», pestaña «Mejoras», «Desenfocar caras»y al finalizar haciendo clic en «Guardar».

c. Creación de códigos QR a través de la dirección web https://www.qrcode-monkey.com/es u otra herramienta similar que permita generar códigos QR.

c.1. Copiar la URL del vídeo de YouTube, haciendo clic en «Compartir» y luego en «Copiar».

c.2. Insertar la URL del vídeo copiado, hacer clic en «Crear código QR» y luego en «Descarga PNG».

d. Es recomendable comprobar los QR descargados en el ordenador empleando una aplicación del dispositivo móvil de lectura de códigos QR: por ejemplo, a través de la app QR Scanner.

3. Maquetación del libro virtual, empleando todos los elementos de la RA.

Figura 2. Maquetación del libro

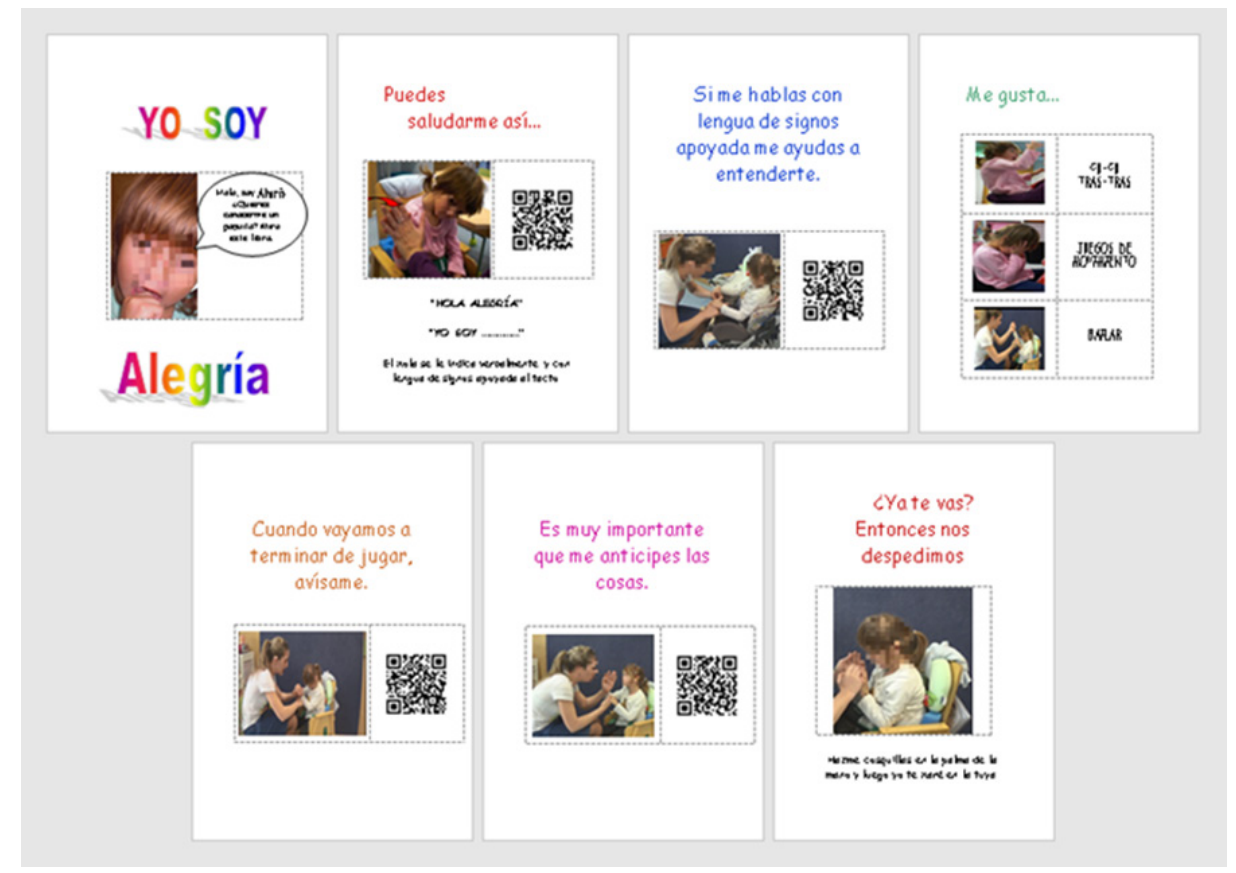

González, M. (2020). Recursos metodológicos que favorecen la intervención comunicativa con niños con sordoceguera utilizando la realidad aumentada: libro de comunicación Yo soy. RED Visual: Revista Especializada en Discapacidad Visual, 76, 24-33. https://oi.org/10.53094/MBMV7925. 
4. Pixelación de las caras con el programa de Windows Paint o cualquier otra herramienta que permita retocar las imágenes.

Si se utiliza el programa Paint, se selecciona la cara a pixelar y con la opción «Cambiar de tamaño y sesgar» se cambian los píxeles de los ejes horizontal y vertical a 10.

5. Montaje del libro físico de comunicación Yo soy.

Se necesitan cartulinas tamaño folio de distintos colores, una anilla para poder sujetarlos en distintos soportes y pegatinas con relieve para decorarlo.

Figura 3. Fotografía del libro físico

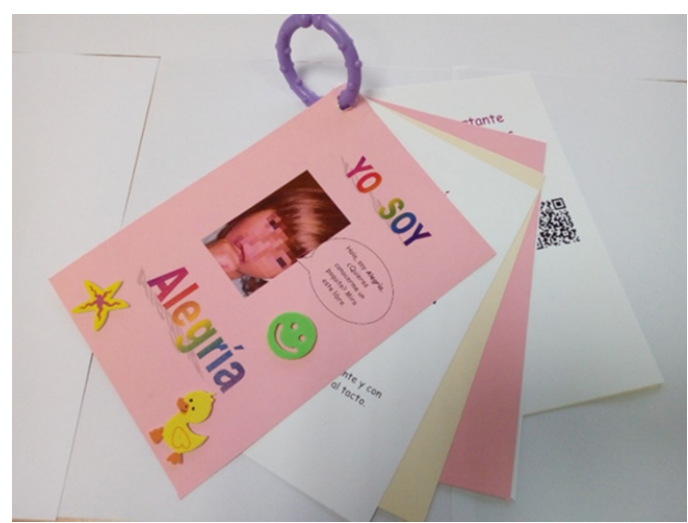

\section{Resultados y conclusiones}

A la hora de evaluar los resultados del libro de comunicación Yo soy se han tenido en cuenta las escalas utilizadas en las observaciones iniciales y las entrevistas realizadas a algunos profesionales, familiares y personas del entorno que lo han utilizado.

En cuanto a los adultos, se muestran satisfechos con el recurso por la sencillez y facilidad de uso. Les da un modelo y seguridad para tener experiencias comunicativas y promover la inclusión en los distintos entornos del niño. Además, un aspecto muy importante que se ha conseguido es la disminución de la percepción de la lengua de signos apoyada como barrera de comunicación.

En cuanto a los niños con sordoceguera congénita, les ha ayudado a mejorar la comprensión del mundo, están más atentos y conectados. También les ha permitido

González, M. (2020). Recursos metodológicos que favorecen la intervención comunicativa con niños con sordoceguera utilizando la realidad aumentada: libro de comunicación Yo soy. RED Visual: Revista Especializada en Discapacidad Visual, 76, 24-33. https://doi.org/10.53094/MBMV7925. 
reducir su aislamiento social con el aumento de las experiencias comunicativas y la disminución de conductas desajustadas al sentirse escuchados y entendidos.

Por todo lo anterior, las familias y profesionales realizan la petición de crear más recursos utilizando la realidad aumentada para poder trabajar estrategias metodológicas en la comunicación con esta utilidad y con esta facilidad de uso.

\section{Referencias bibliográficas}

Gómez, P. (coord.) (2017). Intervención educativa en el alumnado con sordoceguera [PDF]. [Madrid, España]: Ministerio de Educación, Cultura y Deporte. (DOI: http://dx.doi.org/ 10.4438/030-17-124-8).

Gómez, P., y Romero, E. (coord.) (2004). La sordoceguera: un análisis multidisciplinar [Word]. Madrid, España: Organización Nacional de Ciegos Españoles.

Rodbroe, I., y Janssen, M. (2014). Comunicación y sordoceguera congénita, 4 vols. Madrid, España: Organización Nacional de Ciegos Españoles.

Rowland, C. (1990). Matriz de Comunicación. Recurso web: https://communicationmatrix.org/.

Stillman, R., y Battle, C. (1985). Escala Callier-Azusa: Escala para la evaluación de aptitudes comunicativas [Word]. Dallas, Estados Unidos: Centro Callier de Alteraciones de la Comunicación.

Unidad Técnica de Sordoceguera de la ONCE (2010). Directrices generales para la atención de afiliados con sordoceguera. Documento de uso interno sin publicar.

Van Dijk, J. (1968). Movement and communication with rubella children. Conferencia pronunciada en la Annual General Meeting of the National Association for Deaf-Blind and Rubella Children. Sint-Michielsgestel, Países Bajos. Sin publicar.

Milagros González Donate. Especialista en sordoceguera de la ONCE. Delegación Territorial de la ONCE en Aragón. Paseo Echegaray y Caballero, 76; 50003 Zaragoza (España). Correo electrónico: migd@once.es.

González, M. (2020). Recursos metodológicos que favorecen la intervención comunicativa con niños con sordoceguera utilizando la realidad aumentada: libro de comunicación Yo soy. RED Visual: Revista Especializada en Discapacidad Visual, 76, 24-33. https://doi.org/10.53094/MBMV7925. 